This is a post-print copy of the paper which was accepted and published at the Electric systems for aircraft, railway, ship propulsion and road vehicles (ESARS) conference on 3rd March 2015.

http://ieeexplore.ieee.org/xpl/articleDetails.jsp?arnumber $=7101488$

\title{
Development of Voltage Standards for Turbo-electric Distributed Propulsion Aircraft Power Systems
}

\author{
Andrew M. Bollman, Michael J. Armstrong \\ Electrical Power and Control Systems \\ Rolls-Royce North America \\ Indianapolis, IN, USA
}

\author{
Catherine E. Jones, Patrick. J. Norman, Stuart J. \\ Galloway \\ Institute for Energy and Environment \\ University of Strathclyde \\ Glasgow, UK
}

\begin{abstract}
Distributed propulsion is being considered as a possible solution to increase aircraft efficiency, reduce fuel costs and reduce emissions. The size, weight and efficiency of components within a Turbo-electric Distributed Propulsion (TeDP) system are dependent on the voltage level of the electrical power network. Current aircraft voltage standards do not address the architecture of distributed propulsion and so a review of voltage standards from different industries is conducted with areas of commonality addressed. An example of TeDP architecture is presented and analyzed to highlight how current aircraft standards may not apply to TeDP. A summary of challenges in developing standards for a TeDP is compiled with a stakeholder analysis to demonstrate the wide range of industries and personnel with vested interests in the development of voltage standards and recommended practices for TeDP.
\end{abstract}

Index Terms-voltage standards, distributed propulsion, aircraft power network

\section{INTRODUCTION}

Flight-weight propulsive power rated microgrid systems necessitate the introduction of new aircraft distribution system voltage standards. The voltage operating envelope significantly impacts component and system performance. All protection, distribution, control, power conversion, generation, energy storage and thermal management equipment are affected by voltage regulation requirements. Information on the desired nominal voltage and voltage operating range is required to determine nominal and maximum currents for sizing distribution and fault interruption equipment, developing machine topologies and machine controls, and the physical attributes of all component shielding and insulation.

Voltage standards provide assurance that electrical equipment will be operable and integrate effectively by applying generally accepted operating limits that impose requirements on system components. Existing voltage standards express steady-state and transient limits at the consumer interface in order to provide a common framework for component manufacturers. Adherence to the standards ensures that the equipment will operate effectively within the context of a conventional system. The existing voltage standards that only address the power quality at the end of the power line are insufficient to address the unique needs of revolutionary TeDP electrical systems.

Standards are often developed either after products are commercially available or when a product development has reached a high Technical Readiness Level (TRL), hence requiring relevant standards to be revised. Whilst these approaches have potential benefits by not constraining system design, it creates uncertainty when determining what enabling technologies require further development. With the low TRL of TeDP, there is an opportunity to start developing a standard that allows for the optimization of the system, possibly helping drive improvements in enabling technologies. By developing a voltage standard for TeDP systems, the high level system requirements for electrical power for TeDP can start to be defined, which in turn will form a driver for performance requirements and research into enabling technologies.

\section{TEDP ELECTRICAL SYSTEM}

A possible electrical system architecture for a TeDP aircraft, developed for NASA's RTAPS program [1], is shown in Figure 1. It is proposed that this architecture is superconducting, to reach the high power densities required for an aero-electric propulsions system [2]. It utilizes a DC transmission network to decouple the high speed generators and low speed propulsors, and take advantage of the high efficiency of DC superconducting networks. The aircraft features four independent electrical systems. Each electrical system consists of one generator, one AC/DC converter, an associated energy storage device, four DC/AC converters, four propulsors and the associated protection devices. The system can be divided into four distinct regions: generation, transmission, distribution and consumption.

This particular network configuration has been developed to be reconfigurable. This will enable the system to be resilient to the worst case scenario of losing power from one of the two gas turbines, which drive the system generators. In the event of the loss of an engine, the system can be reconfigured such that one engine provides all the necessary power and the power is distributed to all of the propulsors. Therefore, each gas turbine is sized to provide $100 \%$ of the necessary propulsive power (i.e. normally operating at $50 \%$ of its rating), each generator is 
sized to produce $50 \%$ of the total propulsive power and each propulsor motor must account for roughly $7.14 \%$ of the power.

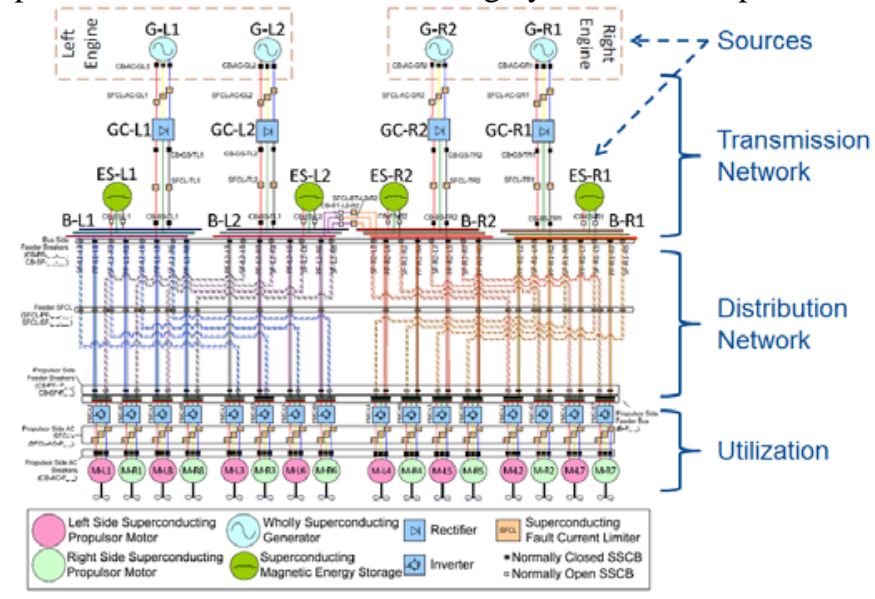

Fig. 1. NASA N3-X RTAPS TeDP Electrical Power Network Example

A TeDP electrical power network is more complex than current electrical power systems for more electric aircraft. It is a compact, islanded microgrid, supplied by a number of generators and energy storage devices. It is not proposed at this stage that the generators load share. The network includes both transmission and distribution networks, featuring both AC and DC power. Finally, the TeDP architecture must have the same, or better, reliability as current aircraft networks. Hence it should be developed with certification in mind. The following short literature review illustrates the different voltage standards from the different existing industries, which may be applicable to TeDP power systems.

\section{REVIEW OF VOLTAGE STANDARDS BY INDUSTRY}

\section{A. Aerospace}

The current standard for voltage on U.S. military aircraft is MIL-STD-704F [3]. This standard is of interest as it addresses both current aircraft voltage standards for both AC and DC systems. AC power is required to be single-phase or threephase with a wire-connected grounded neutral system. While a 400 Hertz, fixed-voltage scheme is typical, the standard allows variable frequency or double voltage equipment. The AC system is regulated using the following characteristics and are defined for both normal and abnormal conditions:

- Steady-state, transient and peak voltage range

- Voltage distortion factor and distortion spectrum

- Voltage unbalance and phase difference

- Voltage modulation

- Voltage recovery times

- DC component

- Power factor

The MIL-STD allows for a two-wire or negative ground return DC system having a nominal voltage of $28 \mathrm{~V}$ or $270 \mathrm{~V}$. The following characteristics are defined to govern the operation of a DC system:

- Steady-state and steady state voltage range
- Distortion factor and distortion spectrum

- Ripple amplitude

- Voltage recovery times

The issue in applying MIL-STD-704 to a TeDP network is that the current standard only applies to the interface of utilization equipment. The generation and distribution of electrical power is left up to the air framer and the only requirement is that it meets MIL-STD-704 at the loads. Hence, there is a clear need for the development of standards which are relevant to aero-electrical systems for TeDP. One option to aid the development of the standards to meet the new requirements imposed by TeDP could be to investigate translating relevant existing terrestrial electric grid standards to future TeDP electrical networks. This would include developing standards which define acceptable voltage levels and power quality throughout the power system: generation, transmission, distribution and load.

\section{B. Bulk Systems and Terrestrial Microgrids}

Bulk power grid standards vary by region and by application. Despite the differences there are common areas of regulation. ANSI C84.1 establishes nominal voltage rating and operating tolerances for $60 \mathrm{~Hz}$ electric power systems above $100 \mathrm{~V}$ and up to and including $1200 \mathrm{kV}$ [4]. ANSI C84.1 takes an approach that establishes the utilization voltage and regulates the voltage drop along the transmission and distribution network. ANSI C84.1 does not closely regulate the high voltage transmission lines due to considerable distances involved, but normally there can be a $+5 \%$ to $-10 \%$ voltage variation from nominal voltage. There are additional voltage drops in the medium and low voltage distribution networks and the National Electric Code assumes a voltage drop at the end consumer. ANSI C84.1 includes two operating ranges, Range $\mathrm{A}$ and Range B. Range A is considered the normal operating range in which all equipment is expected give fully satisfactory performance. ANSI C84.1 Range B includes voltage above and below Range A that results from practical design and operating conditions. When the voltage moves into Range B, corrective measures must be undertaken to improve the voltage to meet Range A requirements and utilization equipment is expected to acceptable performance throughout Range B, although not necessarily as good as performance in Range A.

For disturbances and exceptional conditions, the generating units are required to withstand a temporary drop in voltage without disconnection form the grid and only a small power reduction. Under exceptional conditions, the voltage can drop to near zero for times varying from 250 milliseconds [5] to 150 milliseconds [6] and may depend on the amount of voltage drop [7]. A recovery time limit is specified and a voltage recovery point is dictated after the fault has cleared, usually $90 \%$ of nominal voltage.

Voltage regulation during transient events varies by industry and location. Often the voltage is treated independently but several standards take into account the frequency a unit is operating at. A regional power plant standard in Finland specifies the operating times by voltage and frequency [5]. The British Standards Institute (BSI) has 
created standards for generators running in parallel or isolation [8]. These standards specify a voltage drop for defined load acceptance (35\% to $100 \%$ of rated current) and for loads with power factors between 0.4 and zero lagging.

IEEE 1547 [7] is the standard for interconnecting Distributed Energy Resources (DERs) with electric power system. As many DERs may have converters as a point of interconnect, IEEE 1547 addresses issues specific to switching devices that TeDP may need to consider. The distortion of waveforms is specified as an allowable maximum total distortion with limits for maximum individual harmonics and the direct current injection into the power system also given.

\section{Marine Industry}

\section{1) Maritime Voltage Standards}

Modern maritime vessels are advancing power management systems in several areas that are of interest to TeDP architectures. Both the shipping industry and TeDP are looking at taking advantages of electric propulsion to save energy while investigating different energy storage techniques to power critical loads. Some shipping applications resemble a power grid made up of isolated power generators driving primarily inductive loads such as the pumping motors on a Floating Production Storage and Offloading ship. The shipping industry can provide some insight in isolated microgrids and is already regulated by several societies, including the American Bureau of Shipping (ABS), Det Norske Veritas (DNV) and Lloyd's Register. The regulatory bodies have not created standards for a maritime DC power system for reasons that will be covered in a later section, though progress is being made in that area.

The ABS establishes power requirements for generators, distribution and at the loads [9]. Similar to aircraft and terrestrial standards ABS provide both steady state and transient limits of voltage, recovery times and recovery limits. The ABS transient voltage standards do vary depending on if load is thrown off or thrown on but the load profile is given and is the same for both scenarios. This load profile and voltage regulation helps define the worst case scenario for that system. Finally, the ABS also covers some voltage regulations for energy storage devices, addressing the charge, recharge and discharge rates.

2) Recommendations Practice of Shipboard Power Systems

The Institute of Electrical and Electronic Engineers (IEEE) has developed a recommended practice for power systems on ships [10] and a recommended practice for shipboard electrical installations [11]. The IEEE states that the common ratings of the MVDC power systems, including their operating devices and auxiliary equipment, should be selected from the following:

- $\quad$ Rated maximum voltage

- $\quad$ Rated withstand voltages

- $\quad$ Rated continuous current

- Rated short-time withstand current

- Rated duration of short circuit

The IEEE states that the voltage should be determined by the desired generator voltage, propulsion motor voltage, converter design, load considerations, standard cable ratings, efficiency and arc fault energy. The continuous DC voltage tolerances should be selected considering the normal loads and insulation breakdown while the rated withstand voltage is different for the system than for the power electronics [12]. The choice of withstand voltage allows for different voltage performance criteria or overvoltage patterns. In addition, the degree of exposure to lightning and switching surge overvoltages, the neutral grounding of the system and the overvoltage limiting devices should be considered. Grounding is essential for the MVDC system as the lack of a reference point will allow for the presence of leakage currents that may cause an unpredictable DC offset.

The Quality of Service (QoS) is another factor considered in the MVDC power system of ships. The QoS is the metric of how reliably the power system provides power to the loads. To do this, the loads must be categorized as uninterruptible, shortterm interrupt, long-term interrupt or exempt. This concept is critical for the TeDP system as the loads are providing all of the aircraft thrust and so the classification of any one propulsor, a bank of propulsors or the entire propulsor group may have different classifications.

The power quality may be described in part by the voltage waveform. IEEE Std 45 [11] specifies the harmonic distortion allowed on the system and on the electric propulsion system, setting limits for both total and individual harmonics. IEEE Std 1709 [10] addresses the quality of power on the MVDC bus through voltage ripple and voltage tolerance. It sets the limit on the acceptable RMS value of ripple and noise to be less than 5\% per unit. Also, the following parameters should be defined:

- Maximum non-repetitive peak

- Maximum repetitive peak

- Maximum repetitive peak-to-peak

Finally, it is prudent to note that safety is critical when designing the MVDC power system. There are no known international guidelines for safety of operation of MVDC power systems above $3 \mathrm{kV}$. However, IEEE Std 1628 [13] and MIL-HDBK-1025/10 [14] include safety recommendations that can be applied to a MVDC system. DC arc fault currents will be significant and models that identify the location and severity of arc flash hazard should be developed. The risk of corona discharge is increased with higher voltage and higher current levels, and prevention methods will be necessary.

\section{Challenges in DeVeloping Voltage StandardS FOR TEDP SYSTEMS WITH DC TRANSMISSION AND DISTRIBUTION}

As stated earlier, there exists an opportunity to develop standards that can allow the optimization of TeDP systems. The IEEE has recommended practices established for Medium Voltage DC power systems on ships and it would be beneficial if a similar effort was extended to TeDP systems. Creating TeDP voltage standards should represent an evolution of existing voltage standards. Some of the challenges in creating DC voltage standards in maritime applications will present themselves in created voltage regulation in TeDP systems. System impedances, regeneration and busbar impedance all require careful consideration when designing a DC system [15]. 
Many of the requirements on regulation are derived from the high level system requirements, particularly propulsion requirements and the concept of operations (CONOPS). Fundamentally, for a TeDP system, voltage limits must enable uninterrupted thrust for the aircraft during all flight conditions and operations. This requirement does not specify that the voltage at each propulsor motor must be continuous all the time, rather that no single point failure in the system will lead to a steady state loss in thrust power below the minimum required power requirement. The aircraft thrust will not be greatly affected if the propulsor motor voltage is interrupted for a period of time if the system can recover fast enough, either by restoring voltage to the faulted propulsor motor or by increasing power on the remaining propulsors.

The permissible length of interruption is subject to a further study but will be a major consideration in fault clearance times and recovery times as well as determining the sizing and role of energy storage on the TeDP network. While the permissible voltage interruption time for one propulsor motor can be one length of time, the equivalent permissible time for a bank of propulsors or for the entire system may be different, presumably less. This introduces a concept that while a load type may have one permissible interruption time for an individual failure, the permissible interruption time to multiple loads of that type may be considerably less or even simply just unallowable. Future TeDP aircraft design studies will be required to give more clarity on the impact of losing a propulsor. The requirements about power availability at the propulsors will drive the voltage envelopes, including excursions in exceptional events, transient limits and recovery times.

The TeDP system most likely will need to define voltage standards for each level of the electrical power system, as the voltage standard at the load may be inappropriate for the voltage at the busbar or transmission level. The voltage at the generation, transmission and distribution levels could be defined by voltage drops at the different levels similar to landbased utility networks. An alternative would be to allow the voltage levels to be relaxed at higher levels if there is sufficient reconfigurability in the system, such that the system can route power from a healthy portion of the network to the propulsors faster than it can clear a faulted condition. A third option would be to have tighter upstream regulation, which would make regulation downstream easier. However, such an approach may require additional energy storage and faster fault detection to maintain the voltage within the tighter limits. A combination between utility and aircraft standards may be appropriate where the nominal voltage levels are defined through voltage drops and the voltage clearance times, whilst transient voltage levels and recovery times vary throughout the system.

A TeDP architecture may necessitate a voltage level higher than the voltage currently accepted in aerospace applications. Resolute adherence to Paschen's curve would result in a low voltage system which would result in very high current levels and prohibitively large electrical cables. The industry will have to consider higher voltage levels for TeDP systems and [1] is an example of how the voltage can be chosen to minimize weight and maximize efficiency for a particular architecture. Standards for higher voltage systems in low pressure environments will need to be developed to establish required insulation and distance levels, and possibly the need for pressurized electrical compartments.

Initial studies [1] have identified that improvements in power density are necessary to make TeDP a viable solution to increasing aircraft fuel efficiency. To illustrate this, Figure 2 shows the sensitivity of the weight of the different components which make up one of the four sections of example TeDP architecture shown in Figure 1, to the DC voltage level. The weights shown include the cryogenic cooler. Figure 3 shows the sensitivity of the electrical losses of the different components to the DC voltage levels. These results were generated using a TeDP systems level weight and efficiency sensitivity tool developed by the authors. From these results, and those discussed in [1], the areas for improvement focus on the power electronics and protection equipment and machines. The emergence of key enabling technologies may influence the chosen operating voltage of the network as well as what voltage standards are applied. However, these aspects should not impact on the fundamental requirement of the system, to provide sufficient propulsion power uninterrupted.
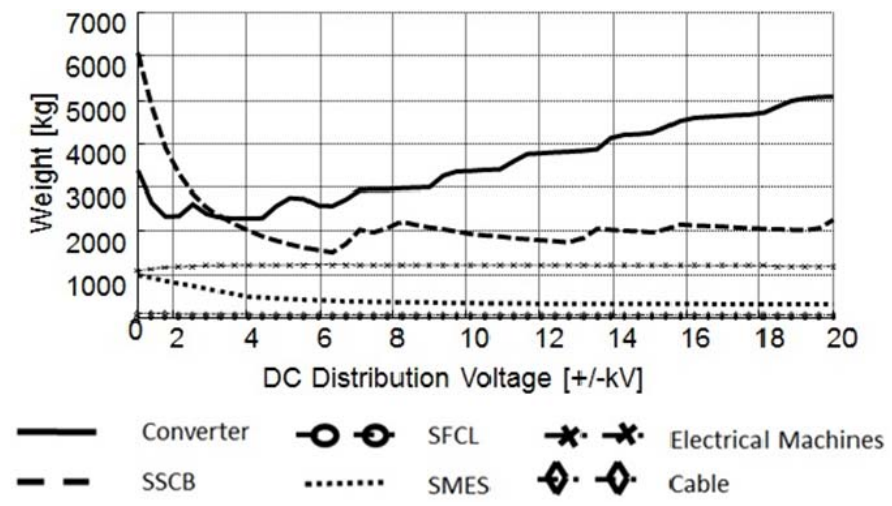

Fig. 2. Sensitivity of the weight of TeDP system components to DC distribution voltage level

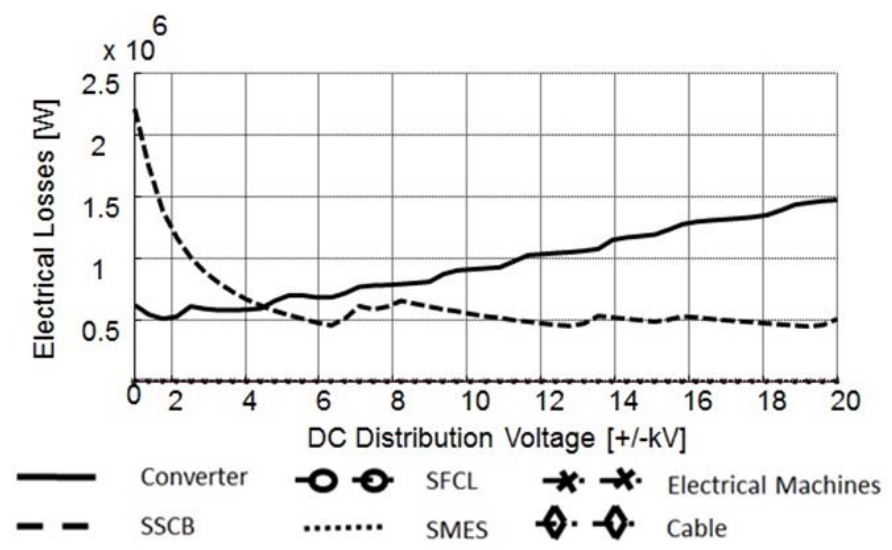

Fig. 3. Sensitivity of the losses of TeDP system components to DC distribution voltage level. 
Whilst this paper has focused on DC systems, trade studies will also be conducted on the viability of AC power systems in TeDP aircraft. Voltage standards will be required for these power systems as well and will provide other challenges to the system and to creating voltage standards. For example, power sharing between buses and between sources is not typical on present-day aircraft but may be allowed in TeDP systems to reduce system size. While power sharing has multiple benefits, it does introduce new failure modes and complicates the design space.

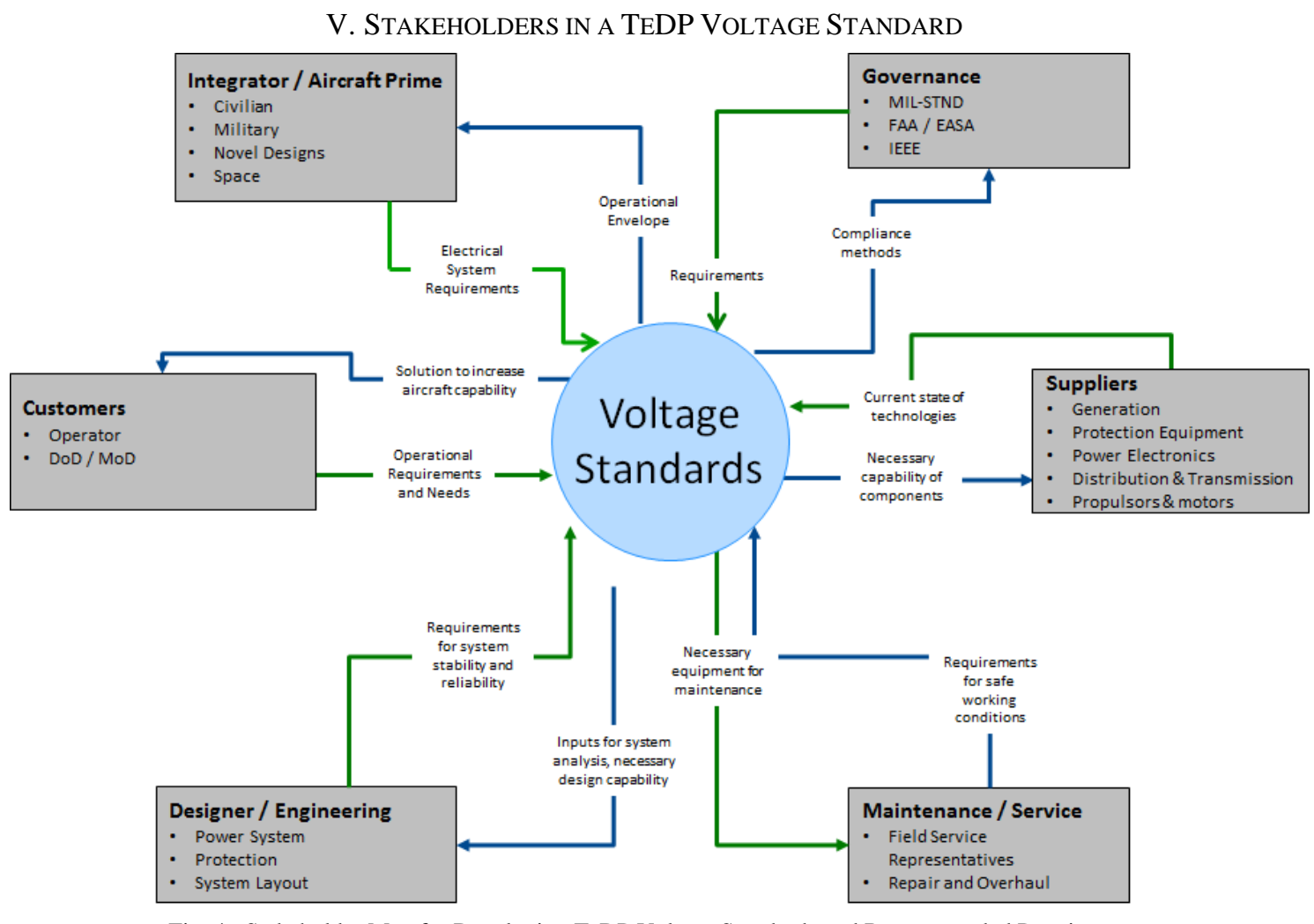

Fig. 4. Stakeholder Map for Developing TeDP Voltage Standards and Recommended Practices

The development of a voltage standard requires addressing a system in multiple perspectives. Therefore, the standard development activity manages stakeholder expectations and visions and results in a common set of bounds which guide the achievement of the goals. These bounds may encompass operational envelope, personnel safety, performance objectives, governance authority or system stability. In order to capture expectations and requirements of different stakeholders, it is important to perform stakeholder analysis. Figure 4 starts to illustrate the major groups that will have input to the voltage standard and what a voltage standard will impact. This study focuses on framing the objectives and sensitivities from a design/engineering perspective.

\section{CONCLUSIONS}

TeDP electrical power networks represent a significant change in aero-electrical systems compared to more-electric aircraft. There is a need to develop standards to ensure the systems are reliable and safe for commercial use. Due to the radical nature of these systems, the current aero-electrical standards are not sufficient.

The development timeline of TeDP aircraft is to be at Technical Readiness Level (TRL) 4-6 in 2025 with entry into service between 2030 and 2035. Hence the possible architecture of a TeDP system is still very developmental. There is a need to develop standards along with the system architecture, redundancy and protection systems, in order to ensure that any aircraft developed can be safe for commercial use.

Parallels between TeDP systems and microgrids in other sections (marine and land) can be drawn, and hence the transferability of standards from those sectors to aerospace requires further investigation and consideration. Finally, further study is required to determine what levels of disturbance, harmonics, variation in voltage and AC frequency level and loss of power supply would be acceptable on a TeDP network.

\section{ACKNOWLEDGMENT}

The material is based upon work supported by the National Aeronautics and Space Administration under Contract Number NNC10BA14B, task order NNC13TA77T. Any opinions, findings, and conclusions or recommendations expressed in this material are those of the author(s) and do not necessarily reflect the views of the National Aeronautics and Space Administration. 


\section{REFERENCES}

[1] M. Armstrong, M. Blackwelder, C. Ross, "Sensitivity of TeDP Microgrid Systems Weight and Efficiency to Operating Voltage," American Institute of Aeronautics and Astronautics, $50^{\text {th }}$ AIAA/ASME/SAE/ASEE Joint Propulsion Conference, July 25, 2014

[2] J.L. Felder, G.V. Brown, H.D. Kim, J. Chu, "Turboelectric Distributed Propulsion in an Hybrid Wing Body Aircraft", International society for Air Breathing Engines Conference, ISABE 2011-1340, 2011

[3] US Department of Defense, "Department of Defense Interface Standard: Aircraft Electric Power Characteristics," MIL_STD_704F, 2004

[4] American National Standard for Electric Power systems and Equipment - Voltage Ratings (60 Hertz), ANSI C84.1-2011

[5] Specifications for the Operational Performance of Power Plants, Pöyry Finland Oy, 30 June 2010, Ref. No 60N50179.10-Fooo6, App. 3.6 Grid Code, Unofficial Translation

[6] Troester, E., "New German Grid Codes for Connecting PV Systems to the Medium Voltage Power Grid,” 2nd International Workshop on Concentrating Photovoltaic Power Plants: Optical Design, Production, Grid Connection

[7] IEEE Standard for Interconnecting Distributed Resources with Electric Power Systems," IEEE Std 1547-2003, pp.1,28, July 28 2003
[8] British Standards Institute General requirements for rotating electric machines - Part 140: Specification for voltage regulation and parallel operation of a.c. synchronous generators, BS 4999-140:1987

[9] American Bureau of Shipping Rules for Building and Classing Steel Vessels, Part 4 Vessel Systems and Machinery, Chapter 8, Section 7: 7, January 12014

[10] IEEE Recommended Practice for $1 \mathrm{kV}$ to $35 \mathrm{kV}$ MediumVoltage DC Power Systems on Ships," IEEE Std 1709-2010, pp.1,54, Nov. 22010

[11] IEEE Recommended Practice for Electrical Installations on Shipboard," IEEE Std 45-2002 (Revision of IEEE Std 45-1998), pp.0_1,258, 2002

[12] IEEE Guide for the Design and Application of Power Electronics in Electrical Power Systems on Ships," IEEE Std 1662-2008, pp.C1,60, March 192009

[13] IEEE Recommended Practice for Maintenance of DC Overhead Contact Systems for Transit Systems," IEEE Std 1628-2009, pp.C1,47, Sept. 252009

[14] US Department of Defense, "Department of Defense Handbook - Safety of Electrical Transmission and Distribution Systems," MIL-HDBK-1025/10, 1998

[15] Butcher, M., Maltby, R., Parvin, P.S., "Compact DC power and propulsion systems - the definitive solution?,” Electric Ship Technologies Symposium, 2009. ESTS 2009. IEEE\ 